LA GRANJA:

REVISTA DE

CIENCIAS DE LA VIDA

pISSN:1390-3799; eISSN:1390-8596

http:/ / doi.org/10.17163/lgr.n26.2017.12
Artículo científico / Scientific paper

CiEnCIAS AgRopecuarias

\title{
USO COMUNITARIO DEL AGUA Y DEL SUELO PARA LA PRODUCCIÓN SUSTENTABLE DE PASTURAS
}

\author{
COMMUNITY USE OF WATER AND LAND FOR SUSTAINABLE PRODUCTION OF \\ PASTURE
}

\begin{abstract}
Charles Jim Cachipuendo Ulcuango*1,3, Narcisa De Jesús Requelme ${ }^{2,4}$, Orlando Marcelo Gualavisí Cachiguango ${ }^{1}$, Catalina Elizabeth Sandoval Montenegro ${ }^{1}$, Jorge Luis Sandoval Cuascota ${ }^{1}$
\end{abstract}

\footnotetext{
1 Universidad Politécnica Salesiana, Grupo de Investigación en Ciencias Ambientales GRICAM, Av. Morán Valverde y Rumichaca, Quito, Ecuador.

2 Universidad Politécnica Salesiana, Centro de Investigación de la Leche CILEC, Av Natalia Jarrín y 9 de Octubre. Cayambe, Ecuador.

${ }^{3}$ Doutorando em Engenharia de Sistemas Agrícolas da Escola Superior de Agricultura "Luiz de Queiroz" Universidade de São Paulo.Dirección: Av. Pádua Dias, 11 - Cx. Postal 9 - Piracicaba - SP, São Paulo, Brasil.

${ }^{4}$ Doctorando en Planificación de Proyectos de Desarollo Rural y Gestión Sostenible, Escuela Técnica Superior Agronómica, Alimentaria y de Biosistemas de la Universidad Politécnica de Madrid, Av. Puerta de Hierro, 2, 28040, Madrid, España

*Autor para correspondencia: ccachipuendo@ups.edu.ec
}

Manuscrito enviado el 17 de marzo de 2017. Aceptado, tras revisión, el 22 de julio de 2017. Publicado el 1 de septiembre de 2017.

\begin{abstract}
Resumen
La ganadería de leche es el principal sistema de producción de las comunidades andinas del Ecuador. La alimentación de los animales se basa en el cultivo de pasturas, que cada día es más difícil por el minifundio de las tierras y cambios en los ciclos de lluvias. Sin embargo, para mantener una producción constante y sustentable, pequeños productores implementan sistemas de riego por aspersión comunitarios que optimizan el uso de los elementos suelo y agua. El objetivo del proyecto fue estudiar el uso de la tierra, eficiencia de los sistemas de riego, y la calidad y salud de suelo como indicadores de sustentabilidad en la producción de pasturas. Se realizaron encuestas a usuarios del riego de la cuenca del río Pisque, se evaluó la eficiencia del riego a nivel de conducción principal, distribución y en parcela y, se tomaron muestras de suelo representativas en todo el territorio. Se observó que hay una estratificación de la tierra según el tamaño de unidades productivas (UPA's) en donde se cultiva pasturas, la eficiencia del uso del agua a nivel de finca es baja con respecto a los otros niveles, y la calidad de los suelos varía según fajas altitudinales. En sí, la producción de pasturas para la alimentación del ganado bovino es realizada en fincas entre 1 a 5 ha y se encuentran en zonas altitudinales de 2850 a $3600 \mathrm{msnm}$, la implementación de sistemas de riego por aspersión y manejo de los índices de calidad de los suelos aportan a la producción sustentable de pasturas.

Palabras claves: Eficiencia riego por aspersión, uso de suelo, cultivo de pasturas, calidad del suelo.
\end{abstract}




\begin{abstract}
Milk livestock is the main system of production of the Andean communities of the Ecuador. Feeding of the animals is based with pastures, that every day is more difficult by the division of the lands "minifundios. ${ }^{\text {and }}$ changes in rainfall cycles. However, to maintain a constant and sustainable production, small farmers implement community systems of sprinkler irrigation, which optimize the use of soil and water. The project aimed to study the land use, efficiency of systems of irrigation, quality, and health of soil as indicators of sustainability in the production of pastures. Surveys to water users were conducted to determine the use of the soil, the irrigation efficiency were evaluated at the level of main channel, distribution and in the plot, and representative soil samples were taken throughout in the territory. It was noted that there is a stratification of the land according to the size of farms, farm-level water use efficiency is low and the quality of soils varies with altitudinal belts. It is concluded that the production of pastures for cattle feeding is done on farms between 1 to $5 \mathrm{ha}$., in the altitudinal zones of 2,850 to 3,600 m. above sea level. The implementation of sprinkler irrigation systems and management of soil quality indices contribute to sustainable pasture production. Keywords: Sprinkler irrigation efficiency; pasture production; land use; quality of soil
\end{abstract}

Forma sugerida de citar: Cachipuendo, C., De Jesús, N., Gualavisí, O., Sandoval, C., Sandoval, J.. 2017. Uso comunitario del agua y del suelo para la producción sustentable de pasturas. La Granja: Revista de Ciencias de la Vida. Vol. 26(2):142-154. pISSN:1390-3799; eISSN:1390-8596. 


\section{Introducción}

El enfoque de la sustentabilidad es una herramienta para la planificación de la producción agrícola en un territorio rural (Senisterra, Gaspari, \& Delgado, 2015), y propende el buen uso del agua y de la tierra entre otros factores. Aguirre (2010), menciona que, desde la perspectiva agroecológica, la ganadería puede ser sustentable si se conjugan elementos que permitan la perdurabilidad en el tiempo de los mecanismos sociales y ecológicos de reproducción del etno-agroecosistema. Entre los principales que anota están: i) la ruptura de las formas de dependencia que ponen en peligro los mecanismos de reproducción, sean estos de naturaleza ecológica, socioeconómica o política; ii) la utilización de recursos que permiten que los ciclos de materiales y energía existentes en el agroecosistema sean lo más cerrados posible; iii) la utilización de los impactos benéficos que se derivan de los ambientes ecológico, económico, social y político existente en y en torno a los sistemas de producción; y, iv) la no alteración sustantiva del medioambiente cuando tales cambios a través de la trama de la vida, pueden implicar transformaciones significativas en los flujos de materiales y energía que permiten el funcionamiento del agroecosistema tolerando y/o aceptando condiciones biofísicas adversas.

La producción de forrajes, como alimento de los hatos ganaderos lecheros es base primordial del desarrollo social y económico del Ecuador (Vera, 2005), pero este sistema de producción demanda considerables recursos como, tierra, suelos fértiles y agua para riego, que en muchas ocasiones son factores limitantes para mejorar la productividad de los pastizales (Martínez Mamian, Ruiz Erazo, \& Morales Velasco, 2016), puesto que, estos recursos forman una trilogía que determina la perdurabilidad del ciclo productivo de forraje en el tiempo (Ates et al., 2013), que, si se logra su equilibrio aportaría a la no dependencia de insumos fuera de la finca para asegurar la producción a lo largo de los ciclos productivos (Aguirre, 2010). Así para este estudio se considera el tamaño de la tenencia de la tierra, la eficiencia del sistema de riego y el índice de calidad y salud de los suelos como indicadores para producción sustentable de pasturas.

El marco normativo constitucional del Ecuador desde el 2008, plantea el paradigma de vida basado en el Sumak Kawsay o "Buen Vivir"donde la naturaleza es sujeto de derechos y la sociedad ecuatoria- na se orienta a vivir en armonía con el entorno natural y no solamente tener las necesidades básicas satisfechas, es decir, existe un marco jurídico que contribuye a que la dimensión ambiental de la sustentabilidad se pueda incorporar a la política pública. Así, el uso de la tierra, manejo del agua y del suelo deben ser enfocados para la producción de alimentos y la generación de condiciones de vida digna en el sector rural (Acosta, 2014).

Para planificar un territorio, toda organización debe implementar un sistema de gestión de información que se encargue de seleccionar, procesar y distribuir la información del uso de la tierra, gestión y manejo del suelo y agua (Aja, 2002), para ello, una herramienta es el "padrón de usuarios" que registra: la propiedad de la tierra, sistemas de producción, gestión del agua, método de riego, uso del suelo y el funcionamiento de las sociedades de regantes organizadas, (Magaz, Hijano, \& Martín, 2008). Los padrones de usuarios están orientados a fines agrarios y dependiendo del enfoque que se quiera dar al análisis de la realidad se ajusta la metodología de aplicación.

El agua es gestionada por las comunidades y organizaciones considerando los principios de equidad y solidaridad (Sandoval Moreno \& Günther, 2013), sin embargo, no se establecen parámetros técnicos de distribución y aplicación del agua en las unidades de producción agropecuaria (UPAs), como: el requerimiento hídrico, la lámina de riego neta según el desarrollo del cultivo, las condiciones climáticas locales y tipo de suelo (Martínez Mamian et al., 2016). En este contexto un indicador de sustentabilidad es, el uso eficiente del agua, determinado por la relación que existe entre los requerimientos hídricos del cultivo y los aplicados con el método de conocido como eficiencia técnica que difiere de la eficiencia económica o conocida también como valoración económica del agua (López Geta, Rubio Campos, \& Martín Machuca, 2005).

Entonces el riego no es sólo infraestructura sino, un conjunto interrelacionado de elementos físicos, ambientales, culturales y socioeconómicos ubicados en un determinado espacio territorial que aprovecha el agua de forma oportuna, en cantidad y calidad para obtener la máxima productividad agropecuaria (Guzmán C., Castro V., JÃEEngwirth M., \& Palenque N., 2002). Por lo que la eficiencia técnica del riego comunitario está estrechamente relacionada con las actividades de gestión y manejo que las organizaciones de regantes implementen (Van Hal- 
sema \& Vincent, 2012), en un sistema de riego según las formas de organización que lo componen se determina la eficiencia a nivel de la parcela, de la comunidad de regantes (distribución) y del canal principal (Playán, 1994).

Otro factor que determina la producción de forraje es el conocimiento de la concentración nutricional de los suelos, el análisis de la fertilidad de los suelos es una herramienta clave en la agricultura sustentable, debe existir un balance nutricional (entradas y salidas), siendo un factor crítico cuando las salidas "cosechas"son mayores que las entradas "nutrientes"generando un déficit nutricional para futuras producciones, por eso, el estudio de los índices de fertilidad conjuntamente con el mapa de pendientes permite definir el uso potencial del suelo (García \& Martínez, 2007). A mediano plazo a través de esta herramienta se generan prácticas agrícolas que consideran las relaciones del suelo, planta, agua y la biota relacionada a la rizósfera (Vivanco, 2011).

El Gobierno Autónomo Descentralizado de la provincia de Pichincha GADPP en su Diagnóstico de Riego y Drenaje (2014) menciona que la actividad agrícola ocupa el 32,5\% de la Población Económicamente Activa (PEA) a nivel rural es la ganadería de leche que cobra importancia como fuente de empleo de la población de las comunidades indígenas y campesinas de la provincia. En este contexto en este artículo se analiza, el uso y tamaño de la tierra, eficiencia de los sistemas de riego e indicie de calidad y salud de los suelos, como indicadores de la producción sustentable de pastizales en el margen izquierdo de la cuenca del rio Pisque

\section{Metodología}

La investigación se desarrolló con 25 organizaciones de riego de las comunidades andinas del margen izquierdo de la cuenca del río Pisque ubicada al norte de la provincia de Pichincha a $75 \mathrm{~km}$ de la capital del Ecuador (Figura 1), este territorio se caracteriza por tener un promedio de temperatura de $13,6 \hat{\mathrm{A}}^{\circ} \mathrm{C}$., una precipitación anual de 519,23 $\mathrm{mm}$, su topografía es ondulada con suelos de origen volcánico, el territorio pertenece al pueblo Kayambi. Las principales actividades productivas con riego son: producción de flores, ganadería de leche y productos para autoconsumo (hortalizas, cereales y tubérculos) (Moreno, 2015).
Para el levantamiento de información se aplicó un enfoque participativo. La participación de los líderes de los sistemas de riego para la generación de la información fue fundamental, y se utilizó la entrevista, encuesta y observación directa como herramientas de investigación.

En el padrón de usuarios se utilizó los sistemas de información geográfica, recorridos de campo y visualización de ortofotos para ubicar geoespacialmente cada una las UPAÂŽs, las fichas de caracterización de las unidades productivas se aplicaron a 5186 regantes conjuntamente con los técnicos de la UPS y líderes de los sistemas de riego; la evaluación de los sistemas de riego se realizó en cada uno de los componentes, midiendo caudales, infiltraciones y evaporación en las captaciones de agua, conducciones y reservorios, a nivel de aplicación del agua en la parcela se evalúo la eficiencia de los aspersores en 27 unidades productivas; finalmente, para establecer los indicadores de fertilidad de los suelos se tomaron 196 muestras a una profundidad de $15 \mathrm{~cm}$ y se analizaron en el laboratorio de suelo de la UPS los siguientes parámetros: $\mathrm{pH}$, materia orgánica, $\mathrm{P}, \mathrm{K}, \mathrm{Ca}, \mathrm{Mg}, \mathrm{Na}, \mathrm{Fe}, \mathrm{Mn}, \mathrm{Cu}, \mathrm{Zn}$, textura, densidad aparente y el contaje de mohos y levaduras. expresados en unidades formadoras de colonias por gramo de suelo seco (UFC/gss).

La información se validó en reuniones con los dirigentes de los sistemas de riego, para el manejo de la información del padrón de usuarios se elaboró una base de datos en el programa informático Access y se ubicaron geoespacialmente las UPAÂŽs utilizando el software ARCGIS.

La necesidad hídrica para el cultivo de pasto se determinó en base a datos de precipitación proporcionadas por el Instituto Nacional de Meteorología e Hidrología (INAMHI), en el período de 10 años (2003-2013) y se utilizó la ecuación de PenmanMonteith, se tomó en cuenta el coeficiente de cultivo (kc) recomendado por la Organización de las Naciones Unidas para la Agricultura y la Alimentación (FAO) (Allen, 2006).

Para determinar la eficiencia del sistema de riego se realizó el análisis a nivel de parcela, de distribución y del canal principal según lo propuesto por Playán (1994). La eficiencia a nivel de parcela ecuación 1 , relaciona las necesidades hídricas que tiene el cultivo y el agua aplicada por el aspersor expresada en porcentaje.

$$
E A=\frac{N H c}{A a s p} \times 100
$$




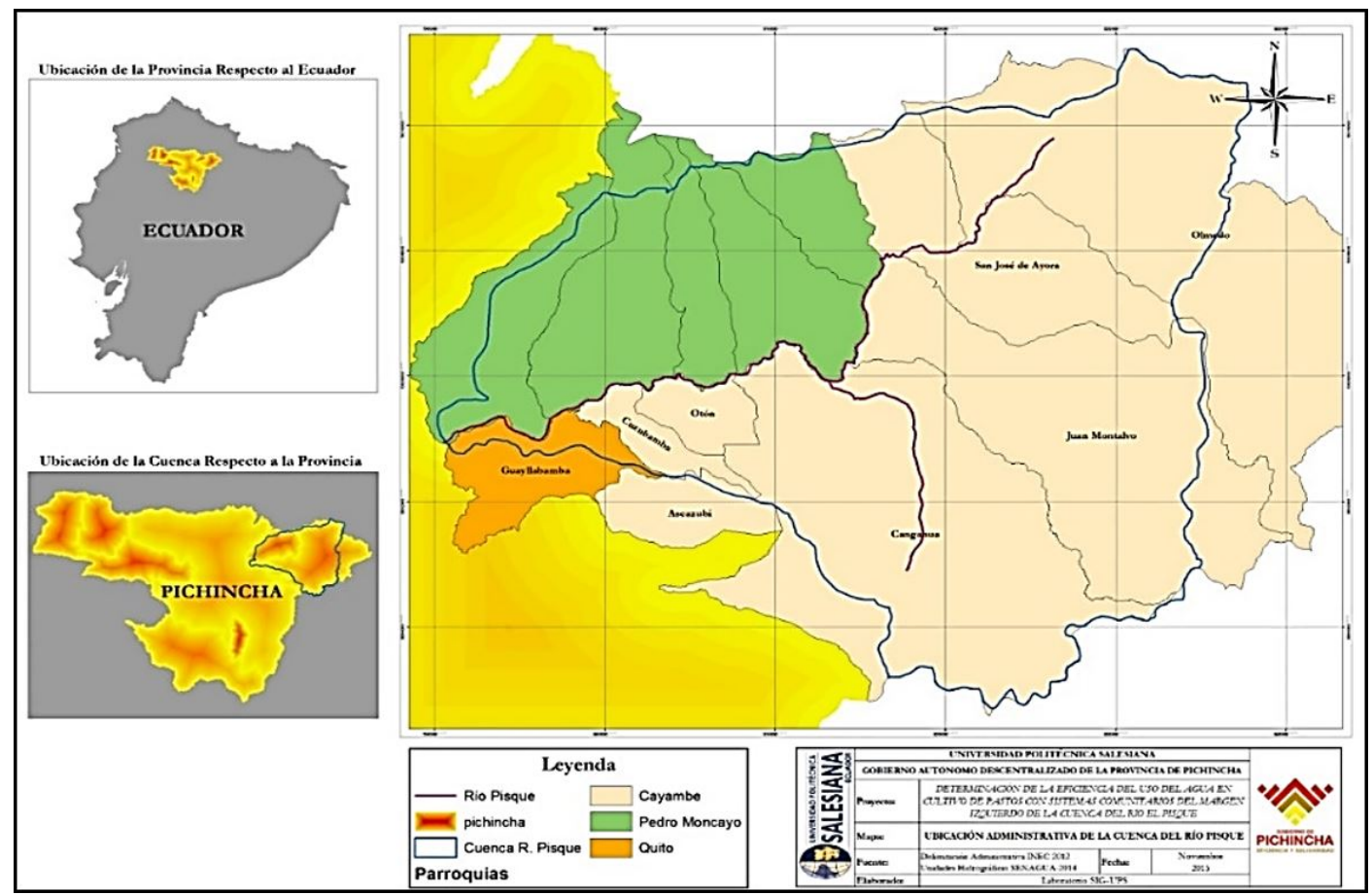

Figura 1. Ubicación Administrativa de la Cuenca del Río Pisque

Donde:

EA = Eficiencia de aplicación a nivel de parcela

$\mathrm{NHc}=$ Necesidades hídricas del cultivo

Aasp = agua aplicada por el aspersor en la parcela

La eficiencia a nivel de distribución se calcula con la ecuación 2, que expresa la relación entre el agua aplicada en la parcela y el agua entregada al sistema.

$$
E D=\frac{\text { Aap }}{\text { Aes }} \times 100
$$

Donde:

$\mathrm{EC}=$ Eficiencia del canal principal

Aes $=$ Agua entregada al sistema

Acp $=$ Agua captada

La eficiencia a nivel de conducción principal es la relación entre el agua captada y la entregada al sistema de riego ecuación 3.

$$
E D=\frac{A e s}{A c p} \times 100
$$

Donde:
$\mathrm{EC}=$ Eficiencia del canal principal

Aes = Agua entregada al sistema

Acp $=$ Agua captada

Para obtener la eficiencia a nivel de todo el sistema se relacionó todos los datos de eficiencia descritos anteriormente según la ecuación 4 .

$$
E S R=\frac{E A}{100} \times \frac{E D}{100} \times \frac{E C}{100} \times 100
$$

Para el caso de los indicadores de fertilidad se realizó el análisis por zonas altitudinales y la valoración de los suelos fue interpolada con los valores fisicoquímico-microbiológicos de los suelos utilizando el software ARCGIS.

\section{Resultados y discusión}

\subsection{Uso del suelo y tenencia de la tierra pa- ra la producción de pasturas}

El Padrón de usuarios constituye una herramienta básica para la gestión del agua de riego, siendo importante que los dirigentes de las comunidades, juntas y/o directorios de agua de riego lo manten- 
gan siempre actualizado, la información principal se basa en la superficie regada, sistemas de producción, métodos de riego y número de usuarios del sistema. A través de la ficha base de riego se ubicaron un total de 6803 fincas y con los SIG se ubicaron 1037 fincas (Tabla 1), distribuidas en 4 categorías por tamaño de finca (971) y 66 corresponden a la superficie ubicada como comunidad o sector. La superficie ubicada con la ficha y los SIG suma un total aproximado de 7.840 fincas ubicadas dentro del margen izquierdo de la cuenca del río Pisque, siendo el $57.3 \%$ de fincas menores de 1 hectárea, es decir, existe un proceso de minifundización del territorio en donde las posibilidades de capitalización de las fincas sean menores y se produzca la migración de los miembros de la familia de las comunidades buscando mejores oportunidades de trabajo principalmente. El minifundio también influye en el diseño, instalación y manejo del riego, este factor puede ocasionar ineficiencia especialmente a nivel de distribución.

En el territorio se identifica la cobertura vegetal natural (páramo, vegetación herbácea, vegetación arbustiva, forestal) y la zona de actividades productivas, diferenciándose la producción agrícola, pecuaria y agropecuaria o mixta (Figura 2).

Tomando como referencia la información obtenida a través de la ficha base de riego, encontramos que el $13 \%$ de las fincas están dedicadas a la producción agrícola, el $41 \%$ de las fincas tienen sistema de producción mixta (agrícola-pecuaria), el 15\% de las fincas tienen sistema de producción pecuaria, y el $31 \%$ de las fincas son suelos que actualmente se encuentran sin cultivar.

En el subsistema agrícola, los productores que destinan parte de la superficie para la producción de alimentos para el consumo familiar y venta del excedente cultivan: papa, fréjol, maíz, cebolla paiteña, col, lechuga, zanahoria, rábano, tomate, hierbas medicinales, arveja, avena, uvilla, cebada, cebolla, chocho, frutilla, habas y quinua. Otros están dedicados al cultivo de flores y la gran mayoría al cultivo de pasto para la producción de leche (Figura 3).

Esta información es relevante en la medida que a partir de ella se pueden desarrollar procesos de diseño, adopción y difusión de sistemas productivos, así como estrategias de manejo de los recursos naturales en un determinado territorio (Torres Lima, Rodríguez Sánchez, \& Sánchez Jerónimo, 2004) orientados a la mayor eficiencia por unidad de producción y no al incremento de animales por super- ficie con parámetros de bajo rendimiento.

\subsection{Carga animal y producción de leche}

La sustentabilidad de los sistemas productivos va de la mano con la intensificación del uso del suelo y tamaño de la tierra, es decir, un número elevado de animales alimentados con el forraje obtenido en una unidad de superficie (ha). Las pasturas andinas son complejas, dependiendo de la altitud y del clima de la zona así como de las especies forrajeras que las conforman la disponibilidad es variable, nótese en la tabla 1 como las fincas $<1$ ha que tienen el mayor porcentaje de pasto mejorado, muestran una carga animal superior que las fincas $>5$ ha con menor porcentaje de pastos mejorados, es decir, el tipo de pastura determina la carga animal de la finca, Requelme, (2012), reporta una carga animal en la zona de $0,54 \mathrm{UBA} / \mathrm{ha}$ en fincas menores de 5 ha tratándose de sistemas extensivos de producción. En términos de producción la relación es inversa, las fincas $>5$ ha con mayor número de vacas en producción, presentan una mayor producción de leche 1/vaca/día mientras que las fincas $<1$ ha con menos animales en producción, tienen un rendimiento menor $(9,8$ 1/vaca/día) pero que en términos de eficiencia en alimentación es superior y en reducción de emisiones de metano es menor contribuyendo así a la sustentabilidad en la producción de estos sistemas de producción de leche.

\subsection{Producción de pastos y necesidades hí- dricas}

La superficie irrigada en el margen izquierdo de la cuenca del río Pisque es de 19 766,98 hectáreas, estando la mayor parte cubierta por el sistema de riego del canal Guanguilqui, que tiene 63 óvalos de distribución y un perímetro irrigado que abarca 4 parroquias, en 43 comunidades y 4 haciendas concienciando con lo expuesto por Acero (2014). Por otro lado, 11 291,36 ha de las tierras agrícolas están dedicadas al cultivo de pasto de estas 7763,18 ha se encuentran con riego por aspersión (Figura 4).

Los meses con presencia de lluvia necesaria para cubrir las demandas del cultivo fueron abril y diciembre con 96,02 y 78,36 mm respectivamente y el mes de menor presencia de lluvia fue agosto, siendo este mes el de mayor demanda hídrica (Tabla 3.), estos meses coinciden con los meses de temperaturas altas y fuertes vientos lo que incrementa la evapo- 
Tabla 1. Número de fincas por tamaño de superficie.

\begin{tabular}{lcccc}
\hline $\begin{array}{l}\text { Categoria de fincas por } \\
\text { superficies }\end{array}$ & $\begin{array}{l}\text { N. de fincas/ } \\
\text { fichas }\end{array}$ & $\begin{array}{l}\text { N. Fincas/ } \\
\text { Ortofotos }\end{array}$ & $\begin{array}{l}\text { N. Fincas } \\
\text { Totales }\end{array}$ & \% Fincas \\
\hline Fincas menores a 1 ha & 4067 & 424 & 4491 & $57,3 \%$ \\
Fincas $>1$ ha hasta $<5$ ha & 2445 & 309 & 2754 & $35,1 \%$ \\
Fincas $>5$ ha hasta $<20$ ha & 275 & 173 & 448 & $5,7 \%$ \\
Fincas $>20$ ha & 16 & 65 & 81 & $1,0 \%$ \\
Comunidades/Sectores & & 66 & 66 & $0,8 \%$ \\
TOTAL & $\mathbf{6 8 0 3}$ & $\mathbf{1 0 3 7}$ & $\mathbf{7 8 4 0}$ & $\mathbf{1 0 0 \%}$ \\
\hline
\end{tabular}

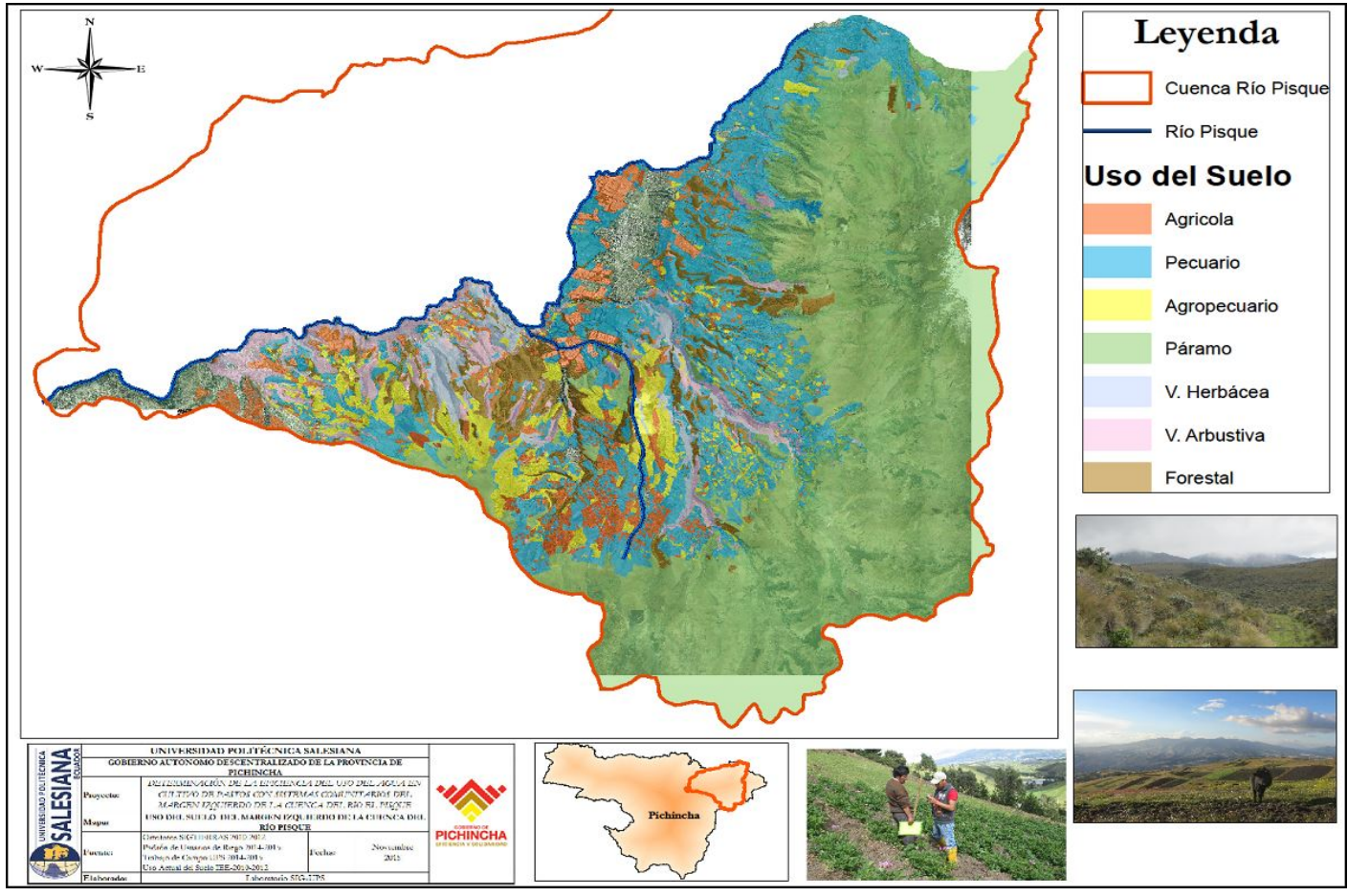

Figura 2. Identificación del uso actual del suelo de acuerdo con las zonas naturales y agrícolas

Tabla 2. Carga animal, tipo de pastura y producción de leche de las fincas por estrato de finca

\begin{tabular}{cccccc}
\hline $\begin{array}{c}\text { Tamaño de } \\
\text { finca (ha) }\end{array}$ & $\begin{array}{l}\text { Carga } \\
\text { animal } \\
\text { UBAs/ha }\end{array}$ & $\begin{array}{l}\text { Número de } \\
\text { vacas } \\
\text { producción }\end{array}$ & $\begin{array}{l}\text { Pasto mejo- } \\
\text { rado }(\mathbf{\%})\end{array}$ & $\begin{array}{l}\text { Pasto } \\
\text { mejorado } \\
(\mathbf{\%})\end{array}$ & $\begin{array}{l}\text { Prod. leche } \\
\text { vaca/dia }\end{array}$ \\
\hline$<1$ & 6,28 & 2,21 & 75,85 & 24,15 & 9,82 \\
$>1<3$ & 4,70 & 3,33 & 77,64 & 22,46 & 9,32 \\
$>3<5$ & 3,76 & 5,22 & 66,31 & 33,69 & 10,47 \\
$>5$ & 3,17 & 8,72 & 69,40 & 30,60 & 11,64 \\
\hline Media & $\mathbf{4 , 7 2}$ & $\mathbf{4 , 2 3}$ & $\mathbf{7 2 , 2 7}$ & $\mathbf{2 7 , 7 3}$ & $\mathbf{1 0 , 3 1}$ \\
\hline
\end{tabular}




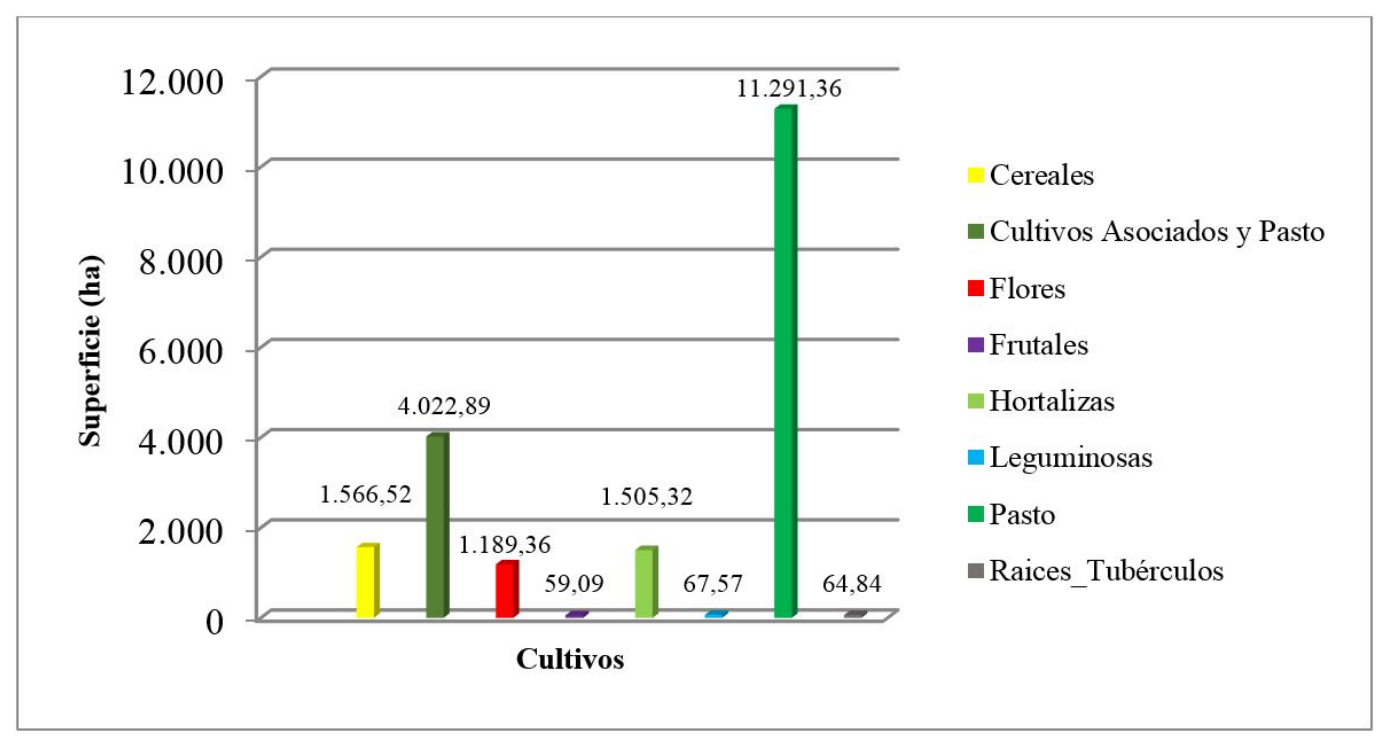

Figura 3. Superficie por tipo de cultivos en el margen izquierdo de la cuenca del río Pisque

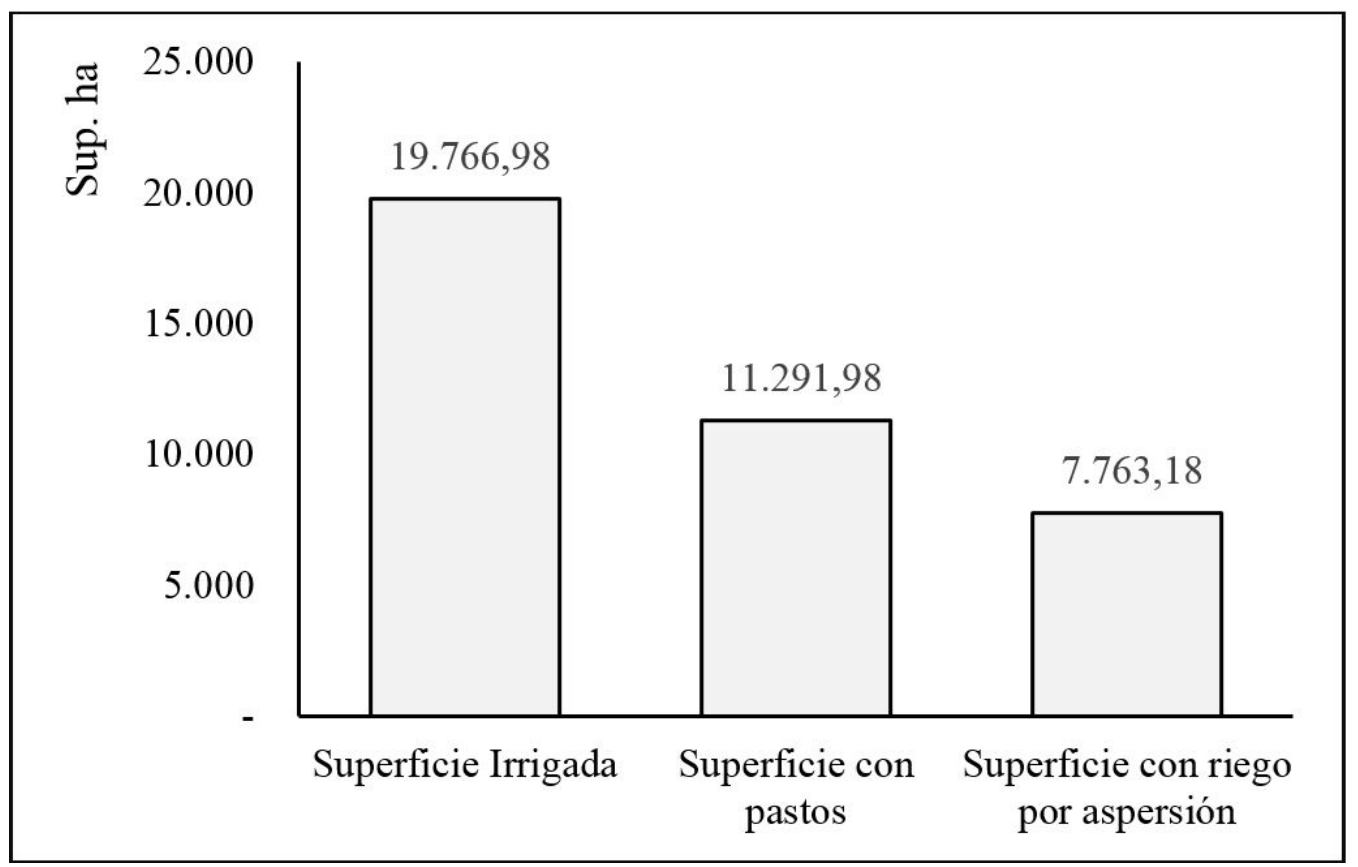

Figura 4. Superficie irrigada y producción de pastos con riego por aspersión 
Tabla 3. Necesidades hídricas para el cultivo pasto

\begin{tabular}{lcccr}
\hline \multirow{2}{*}{ Mes } & P mes mm & \multicolumn{4}{c}{ Necesidades hídricas en m3 } \\
\cline { 3 - 5 } & & ha & día & mes \\
\hline Enero & 47,36 & 25,33 & $286.042,27$ & $8.867 .310,31$ \\
Febrero & 62,04 & 19,63 & $221.692,93$ & $6.429 .095,09$ \\
Marzo & 72,26 & 16,94 & $191.267,69$ & $5.929 .298,43$ \\
Abril & 96,02 & 8,83 & $99.685,60$ & $2.990 .568,02$ \\
Mayo & 48,87 & 25,04 & $282.744,26$ & $8.765 .072,17$ \\
Junio & 29,45 & 28,94 & $326.799,33$ & $9.803 .979,94$ \\
Julio & 16,85 & 31,24 & $352.724,43$ & $10.581 .732,79$ \\
Agosto & 10,78 & 35,74 & $403.575,06$ & $12.510 .826,88$ \\
septiembre & 23,19 & 36,50 & $412.079,89$ & $12.362 .396,82$ \\
Octubre & 72,25 & 19,62 & $221.575,56$ & $6.868 .842,23$ \\
noviembre & 69,84 & 19,07 & $215.281,75$ & $6.458 .452,62$ \\
diciembre & 78,36 & 26,26 & $296.558,80$ & $9.193 .322,66$ \\
\hline
\end{tabular}

transpiración y las necesidades hídricas de los cultivos son mayores, datos que coinciden con los reportados por Sandoval (2014).

Para suplir este déficit y mantener la producción de pasturas de forma constante durante todo el año, las organizaciones de regantes impulsan la presurización de los sistemas de riego aprovechando la energía producida por la fuerza de la gravedad, y utilizan el método de riego por aspersión semifijo para aplicar el agua en la parcela. En este contexto en el margen izquierdo de la cuenca del río Pisque la superficie es regada a través de tres métodos, cerca del $61 \%$ de la superficie total es regada por aspersión, el $26 \%$ riega por inundación y solo $1 \%$ riega por goteo, el $12 \%$ restante de la superficie no tiene riego a nivel de parcela. La implementación del riego por aspersión en territorios comunitarios permite optimizar el uso del agua en la producción agrícola (Communal, Faysse, Bleuze, \& Aceldo, 2016), permitiendo incrementar la sustentabilidad del territorio.

\subsection{Eficiencia de sistemas de riego comuni- tario}

Entre los factores que afectan la eficiencia del sistema de riego por aspersión comunitarios se encuentran: el tamaño de los perímetros irrigados, método de riego y el manejo del sistema. Con características topográficas onduladas, inclinadas e la influencia del viento, la eficiencia de riego a nivel de parcela es del $38.72 \%$ (Tabla 4 ) siendo muy bajo con los repor- tados por otros autores que para el riego por aspersión oscila entre $70 \%$ y $80 \%$ (Cisneros, Pacheco, \& Feyen, 2007). La baja eficiencia, se atribuye al viento, accesorios deteriorados, mangueras sin sus respectivos acoples y aspersores en mal estado (boquillas modificadas) etc., otro factor que influye enormemente es el tiempo de riego por cada posición del aspersor, es decir, se aplica más agua de lo que requiere la planta; y, el marco de riego es variable porque los equipos móviles no siempre permiten tener una distancia fija en los traslapes. El promedio de la eficiencia a nivel de distribución es del 74.54\% (Tabla 4), y es influenciada por los daños que existen en el trayecto desde los hidrantes hasta los aspersores donde se desperdicia agua y no se está regando con todo el volumen de agua entregado al sector por cada uno de los turnos de riego o a su vez la cantidad de aspersores que riegan no son suficientes, es decir que el factor mientras mayor sea el perímetro irrigado menor es la eficiencia. En este componente el agua se encuentra presurizada por lo también influye en la eficiencia por las pérdidas de carga que se producen en las tuberías de conducción. La eficiencia promedio a nivel de conducción principal es del $90 \%$ (Tabla 4), debido principalmente a que la conducción principal desde la captación hasta el reservorio, está totalmente entubada en la mayoría de los sistemas evaluados, razón por la cual "todo. ${ }^{\text {el }}$ caudal de agua captado llega al lugar de almacenamiento; pero también existen sistemas que están bajo la media y se debe a que la conducción principal es a canal abierto y en el trayecto desde la captación 
hasta el lugar de almacenamiento se pierden volúmenes de agua por robos, evaporación, filtraciones, y por la vegetación que se encuentra cerca de los canales. La eficiencia media a nivel general de los sistemas de riego evaluados es del $30.49 \%$ (Tabla 4), siendo una eficiencia baja con los resultaos reportados por Shahbaz (2006), que es alrededor del $50 \%$, y del $54 \%$ reportado por Uzen (2016), siendo el valor óptimo el $100 \%$, tomando en cuenta la cantidad de agua captada versus la cantidad de agua aplicada a la planta, pero es difícil obtener la perfección especialmente en los sistemas de riego comunitarios andinos en donde predominan factores físicos, climáticos y principalmente humanos. En factores los físicos se pueden señalar las pendientes fuertes hasta del $45 \%$, el tipo de suelo que varía de arenoso, franco y arcilloso con sus respectivas combinaciones y el tipo de infraestructura que en sus diseños solo consideran los criterios hidráulicos dejando un lado los criterios agronómicos y principalmente sociales (Playán \& Mateos, 2006). El viento que en las partes altas de la cuenca alcanza hasta $40 \mathrm{~km} \cdot \mathrm{h}^{-1}$ influye en la eficiencia a nivel de parcela principalmente y finalmente el factor más influyente en la eficiencia es el factor social que depende de las acciones humanas individuales y colectivas.

\section{5 índices de calidad de los suelos dedica- dos a la producción de pastizales}

La valoración de los índices de calidad y salud de los suelos se realiza en el área comprendida entre los 2 800-3 $600 \mathrm{msnm}$, en este rango altitudinal se detectó la mayor superficie de suelo destinada a producción de pastizales. En la Tabla 5, se observa que los suelos dedicados a la producción de pastos sobre los 3300 a $3600 \mathrm{msnm}$, presentan los más altos índices de calidad de los suelos. Esto indica que la implementación de prácticas agrícolas desarrolladas en suelos sobre los $3300 \mathrm{msnm}$, no han provocado de manera significativa efectos negativos sobre las propiedades físicas y químicas. Por otro lado, al reducir la altitud de la zona en estudio, los índices de calidad y salud de suelos disminuyen, sin embargo, los mencionados índices se encuentran sobre los valores considerados como adecuados para los suelos agrícolas. Si bien existe diferencia en relación con el índice de calidad y salud de los suelos entre fajas altitudinales se puede atribuir esta diferencia al manejo agrícola del suelo (Communal et al., 2016).
La valoración de los índices de calidad y salud de los suelos obtenidos en la zona en estudio, demuestran que las características físicas, químicas y microbiológicas de los suelos analizados son óptimas y que las actividades agrícolas desarrolladas en el sector no han logrado alterar la composición nutricional de los suelos ni alterar su dinámica microbiológica, puntualmente, sobre el contaje de unidades formadoras de colonias por gramo de suelo seco (UFC/gss) de mohos y levaduras diagnosticado. Esto último es ratificado por Gallardo (2012), quien manifiesta que los valores esperados de mohos y levaduras en suelos de trópico alto saludables, debe ubicarse entre 1x106 (UFC/gss), y los valores microbiológicos encontrados en esta investigación fluctúan sobre este valor. Una condición que cuidar sobre los suelos cultivables es fomentar la incorporación de microorganismos al suelo que a corto plazo contribuyan en la asimilación de nutrientes por parte de las plantas.

\subsection{Producción potencial de pastos}

Con los criterios de disponibilidad riego, Figuras de pendientes e índices de calidad y salud de los suelos se elabora el Figura de uso potencial del suelo para la producción de pastos en el margen izquierdo de la cuenca del río Pisque, dando como resultado que una superficie 14797.75 ha, sería lo más recomendable para este territorio (Figura 3). Para una producción agrícola sustentable el factor determinante es dotar de agua de riego de forma oportuna y eficiente (Communal et al., 2016).

\section{Conclusiones}

La producción de pastizales en esta zona andina se realiza mayoritariamente en unidades productivas que oscilan de 1 a 5 ha., donde los índices de calidad y salud de los suelos son altos, y los agricultores mediante procesos de innovación sin costo de energía implementan sistemas de riego por aspersión aprovechando la fuera de la gravedad.

La eficiencia de los sistemas de riego difiere según los niveles, la mayor ineficiencia se presenta a nivel de aplicación del agua en la parcela, principalmente por desconocimiento del regante en el manejo de los aspersores y láminas de agua; a nivel de distribución la eficiencia es alta porque la mayoría de sistemas de riego cuentan con reservorios y redes 
Tabla 4. Eficiencia promedio del riego por aspersión según sus niveles

\begin{tabular}{lc}
\hline \multicolumn{1}{c}{ Eficiencia por niveles } & Porcentaje \\
\hline Parcela & 38,72 \\
Distribución & 74,54 \\
Conducción principal (canal) & 90,04 \\
Promedio general de eficiencia de los sis- & 30,49 \\
temas de riego evaluados & \\
\hline
\end{tabular}

Tabla 5. índices de calidad y salud de los suelos

\begin{tabular}{|c|c|c|c|}
\hline \multirow{2}{*}{$\begin{array}{l}\text { Fajas } \\
\text { Promed } \\
\text { msnm } \\
\end{array}$} & \multirow{2}{*}{ Altitudinales } & \multicolumn{2}{|c|}{$\begin{array}{l}\text { Indices de calidad y } \\
\text { salud de los suelos }\end{array}$} \\
\hline & & (f) & $(\%)$ \\
\hline & 2.897 & 0,66 & $66 \%$ \\
\hline & 3.105 & 0,70 & $70 \%$ \\
\hline & 3.314 & 0,80 & $80 \%$ \\
\hline & 3.480 & 0,81 & $81 \%$ \\
\hline & 3.638 & 0,85 & $85 \%$ \\
\hline
\end{tabular}

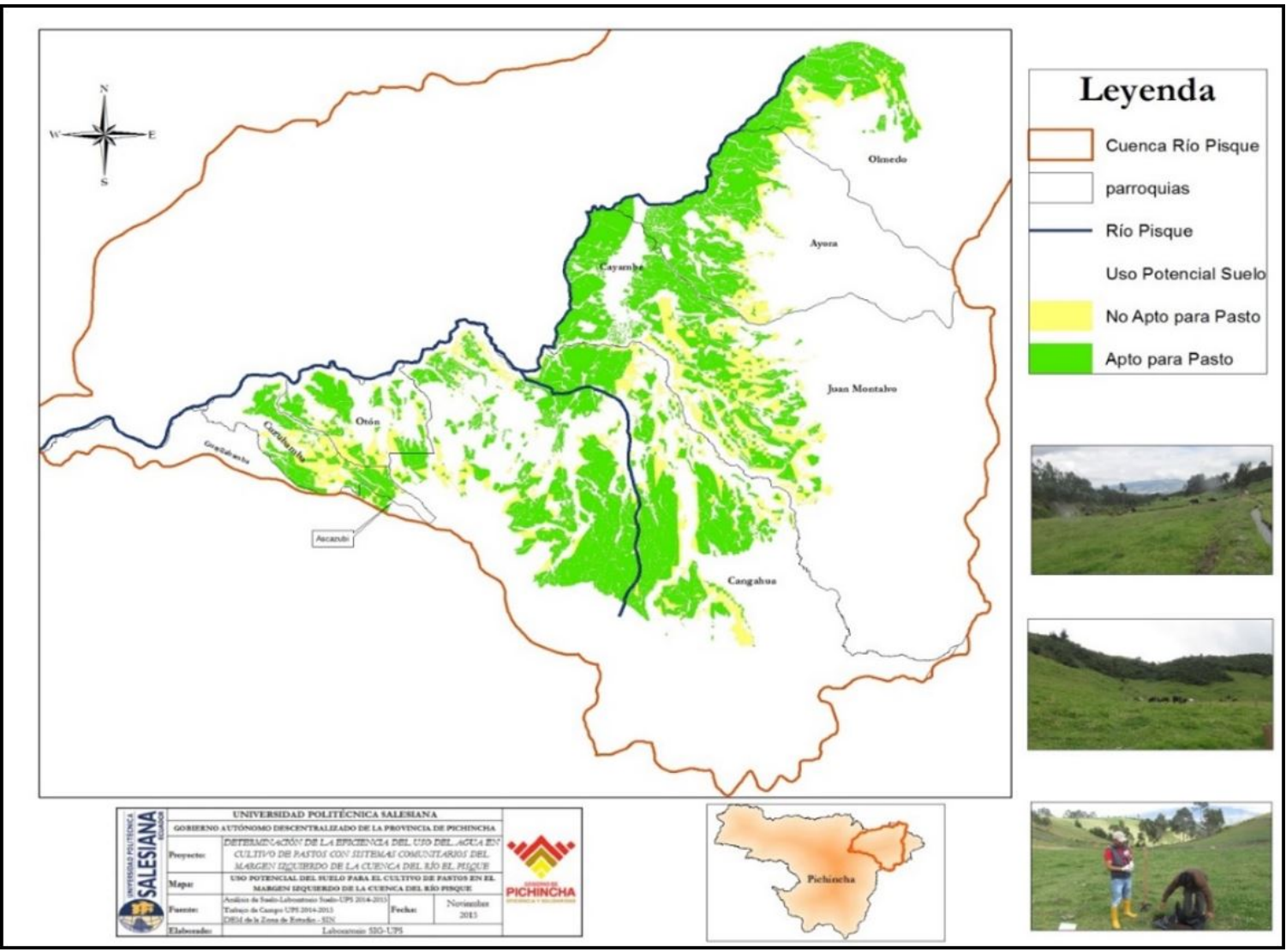

Figura 5. Uso potencial del suelo para el cultivo de pastos 
de conducción secundaria y terciarias entubadas; y, a nivel de canal principal tienen una alta eficiencia debido a que los sistemas de conducción de la fuente hasta los reservorios son entubados o canales revestidos.

Los índices de calidad y salud de los suelos permiten correlacionar las variables de producción de pastos sobre la conservación de los suelos, presentándose mejores índices en las fajas altitudinales próximas a la frontera o límite agrícola (sobre los $3000 \mathrm{msnm}$ ), principalmente, por los altos contenidos de materia orgánica. Los bajos valores de $\mathrm{pH}$ y conductividad eléctrica entre otros, en fajas altitudinales por debajo de los $2900 \mathrm{msnm}$, presentan un $\mathrm{pH}$ ligeramente neutro y un menor contenido de materia orgánica por lo que los indicadores bajan, evidenciado en el sector un efecto erosivo de los suelos, también estos suelos presentan un mayor contenido de arena lo que permite contar con suelos de clase textural franco arenosos arena. El porcentaje de arena sobre los suelos disminuye en la medida que incrementa la altitud y debe ser considerar al momento de manejar el riego. Si bien los niveles de fertilidad van de moderados a intermedios para la producción de pasturas es necesario concienciar a los productores sobre la importancia de restituir los nutrientes, posterior a la cosecha de pastizales.

La participación de los sujetos de investigación genera confianza en el levantamiento de información y al mismo tiempo se generan habilidades y destrezas en la gestión y manejo de los sistemas de riego y del suelo, así el padrón de usuarios permitió conocer el número de usuarios, la superficie regada, sistemas de producción y métodos de riego, de manera que las decisiones se fundamenten con información, social, ambiental, económico y tecnológica de manera que la producción de pasturas tenga mayor sustentabilidad en el territorio. Sin embargo, resta por estudiar el valor nutritivo de especies forrajeras nativas a fin de trabajar balances forrajeros que mantengan niveles de producción y reproducción óptimos y aporten a la sustentabilidad de la actividad ganadera.

\section{Referencias}

Aguirre De Juana, á. 2010. Sostenibilidad en explotaciones agropecuarias de montaña. Escuela Técnica Superior de Ingenieros Agrónomos. Universidad Politécnica de Madrid. España.
Disponible en: https://goo.gl/rp2gJN

Acero, J. 2014. Identificación geoespacial, organizativa y agropecuaria del canal de riego Guanguilquí. Universidad Politécnica Salesiana. Ecuador. Disponible en: https://goo.gl/ UTPXzi

Acosta, A. 2014. El Buen Vivir como alternativa al desarrollo. Algunas reflexiones económicas y no tan económicas. Política y Sociedad, 52(2), p. 299-312. DOI: http://dx.doi.org/10.5209/rev_ POSO.2015.v52.n2.45203

Allen, R., Pereyra, L., Raes, D., Smith, M. 2006. Evapotranspiración del cultivo: Guías para determinación los requerimientos de agua de los cultivos. (FAO). Disponible en: https:// goo.gl/F9eALU

Ates, S., Isik, S., Keles, G., Aktas, A. H., Louhaichi, M. y Nangia, V. 2013. Evaluation of deficit irrigation for efficient sheep production from permanent sown pastures in a dry continental climate. Agricultural Water Management, 119, p. 135-143. DOI: https://doi.org/ 10.1016/j.agwat.2012.12.017

Cisneros, F., Pacheco, E. y Feyen, J. 2007. Evaluación del rendimiento de sistemas de riego por aspersión de baja pluviosidad como resultado de la aplicación de la extensión como soporte técnico. Ingeniería Del Agua, 14(3), p. 177-186. Disponible en: https:/ /goo.gl/2GAwVt

Communal, T., Faysse, N., Bleuze, S. y Aceldo, B. (2016). Effects at Farm and Community Level of the Adoption of Sprinkler Irrigation in the Ecuadorian Andes. Irrigation and Drainage, 65(4), p. 559-567. DOI: https://doi.org/10. 1002/ird.2063

Dirección de Gestión del Riego de la Provincia de Pichincha 2014. Diagnóstico de Riego y Drenaje de la Provincia de Pichincha. Quito.

Gallardo, J. 2012. Metodologías de estudio de la materia orgánica del suelo. La materia orgánica edáfica: Metodologías de estudio. Instituto de recursos naturales y agrobiología de Salamanca. p. 13-15.

García, H. y Martínez, G. 2007. Uso de ortofotos para actualizar el mapa de uso del sue- 
lo en Guanajuato, México. Agricultura Técnica En México, 33(3), p. 271-279. Disponible en: https://goo.gl/mxuXbw

Guzmán C., R., Castro V., M., Jüngwirth M., J. y Palenque N. W. 2002. Del proceso de acompañamiento hacia la autogestión de sistemas de riego. In S. Cáceres (Ed.), (entrelínea, p. 2). La Paz - Bolivia: Editorial Offset Boliviana Ltda. Disponible en: https://goo.gl/FRgaVN

López Geta, J. A., Rubio Campos, J. C. y Martín Machuca, M. 2005. VI Simposio del agua en Andalucía. Sevilla - España: Ibergraphi 2002. Disponible en: https://goo.gl/RmBNnj

Magaz, J. C. M., Hijano, C. F. y Martín, J. A. G. 2008. Los padrones de riego de Argamasilla del Alba (Ciudad Real): un documento paracatastral regulando los derechos al agua en los siglos XVIII y XIX. CT: Catastro, 63, p. 181-201. Disponible en: https://goo.gl/mTxSJS

Martínez Mamian, C. A., Ruiz Erazo, X. A. y Morales Velasco, S. 2016. Huella Hídrica De Una Finca Ganadera Lechera Bajo Las Condiciones Agroecológicas Del Valle Del Cauca. Biotecnología en el sector agropecuario y agroindustrial, 14(2), p. 47-56. DOI: http://dx.doi.org/ 10.18684/BSAA(14)47-56.

Moreno, L. 2015. Actualización del plan de desarrollo y ordenamiento territorial del cantón Cayambe. Cayambe.

Playán, E. 1994. Eficiencia en el aprovechamiento de agua por regadío. Geórgica: Revista del Espacio Rural, 3, p. 99-128. Disponible en: https: //goo.gl/htuUjF

Playán, E. y Mateos, L. 2006. Modernization and optimization of irrigation systems to increase water productivity. Agricultural Water Management, 80(1-3 SPEC. ISS). Disponible en: https://goo.gl/rFnZmf

Requelme, N. y Bonifaz, N. 2012. Caracterización de sistemas de producción lechera de Ecuador. La Granja: Revista de Ciencias de la Vi- da, 16(2), p. 23-47. DOI: http://dx.doi.org/10. 17163/lgr.n15.2012.05

Sandoval, J. 2014. Necesidades hídricas de pasturas con manejo orgánico en la zona de influencia del canal de riego Cayambe - Pedro Moncayo. Universidad Politécnica Salesiana. Disponible en: https://goo.gl/ZNX58H

Sandoval Moreno, A. y Günther, M. G. 2013. La gestión comunitaria del agua en México y Ecuador: otros acercamientos a la sustentabilidad. Ra Ximhai, 9, p. 165-179. Disponible en: https://goo.gl/2soJA9

Senisterra, G. E., Gaspari, F. J. y Delgado, M. I. 2015. Zonificación de la vulnerabilidad ambiental en una cuenca serrana rural, Argentina. Revista Estudios Ambientales, 3(1) p. 38-58. Disponible en: https://goo.gl/aYp5nh

Torres Lima, P., Rodríguez Sánchez, L. Sánchez Jerónimo, ó. 2004. Evaluación de la sustentabilidad del desarrollo regional. El marco de la agricultura. Región y Sociedad, XVI. 16(29), p. 1-36. Disponible en: https://goo.gl/GB9Erb

Uzen, N. y Cetin, O. 2016. Evaluation of physical and economical water use indices in two irrigation schemes in southeastern Anatolia region of Turkey. Fresenius Environmental Bulletin, 25(11), p. 4863-4871. Disponible en: https://goo.gl/BfoF8Y

Van Halsema, G. E. y Vincent, L. 2012. Efficiency and productivity terms for water management: A matter of contextual relativism versus general absolutism. Agricultural Water Management, 108, 9-15. DOI: https://doi.org/10. 1016/j.agwat.2011.05.016

Vera, R. 2005. Perfiles por País del Recurso Pastura / Forraje Ecuador. Sierra, 392, 1-57.

Vivanco, J. M. 2011. El ambiente rizosférico, el mundo oculto de la productividad agrícola. DIHP, Intagri. Celaya, México. p. 3. Disponible en: https:/ /goo.gl/u6wcKf 